\title{
The research of remote sensing image fusion technology
}

\author{
Wang Bin \\ College of Information Science \& Electronic \\ Technology of jiamusi university \\ Jiamusi,china \\ e-mail: jmsuwang@163.com
}

\author{
Wang Chao* (Author for correspondence) \\ College of Information Science \& Electronic \\ Technology of jiamusi university \\ Jiamusi,china \\ e-mail: shuzi0920@163.com
}

\begin{abstract}
Remote sensing image fusion is an important branch in the field of image fusion. Remote sensing image fusion mainly researches how to use different aerial remote sensor to obtain relevant image information. In view of the present research status in this field and the full analysis of remote sensing image fusion method and wavelet transform, the paper aims at proposing the image fusion technology of combining discrete multiwavelet transform and HIS method, then the paper analyses the experimental results with the help of MATLAB. It is proved that the analysis method of wavelet has more advantages than traditional methods: Wavelet transform fusion method can keep maximized resolution and multispectral image.
\end{abstract}

Keywords-Remote sensing; Fusion; Wavelet ;Transform

\section{INTRODUCTION}

Remote sensing technology is a comprehensive technology for Earth Observation. It appears to meet the objective need of the people. Compared with other technologies, it has incomparable technical advantage. Information fusion of remote sensing image processes many remote sensing images of the same target by using certain data algorithm to generate a new and clearer image in a unified coordinate system. Remote sensing data have different temporal resolution, spatial resolution and Popper's resolution in different band. In order to get high-definition remote sensing image, we can adopt some fusion algorithm.

With the improvement of science and technology, the modern remote sensing technology also has rapid development. Earth satellite continuously provides a large number of images with different spectral resolution, temporal resolution and spatial resolution. Due to a variety of reasons of design and observation, time, spatial and spectral of the images provided by different remote sensing data have great difference. In order to recognize and understand the target more clearly, comprehensively and precisely, people try to use a method that can comprehensively utilize all kinds of data images. That is the reason for the birth of fusion technology of remote sensing image.

In the field of image fusion technology, wavelet transform fusion is the focus of research in recent years, and the achievements are remarkable. The further expansion of unit-wavelet is multiwavelet, multiwavelet has many good characteristics, for example: orthogonality, symmetry, Two Vanishing/2-order vanishing, Two Vanishing Moments and short supports. It is due to a variety of advantages, it can provide image fusion method of high resolution good accuracy.

This study aims at combining HIS with multiwavelet transform, and the study is based on previous research and the comprehensive analysis to the characteristics of multiwavelet transform and remote sensing multi-source data fusion method. Through this method, the fusion tests were conducted on multispectral images and panchromatic images with different resolution, and all the images were gotten from different sensors. The experimental results show that wavelet analysis method has remarkable advantages.

\section{THE BASIC PRINCIPLE OF THE METHOD OF WAVELET TRANSFORM FUSION}

Remote sensing image fusion is essentially image synthesis technology. According to certain algorithm, spectral resolution images that gotten from different sensors or different platform are processed to get high quality, clear and accurate pictures. The key of image fusion algorithm is to process the redundant or complementary data in time or space with certain algorithm so as to obtain relevant information of the image, and to create a composite image which has new characteristics in space, spectrum, and time. The image fusion technology not only realizes the simple conformity of image, but also realizes the optimization of image information.

The concept of Multiresolution analysis is put forward by Mallat in 1989 when he structured orthogonal wavelet base. This concept presents more specific analysis and explanation to the characteristics of multiresolution of the wavelet from the perspective of space. The specific principles of the model are as follows: Assume to decompose the image into L layer, $(31+1)$ layer subbands, including the low frequency band $C_{j}$ and the high frequency subbands $D^{h} 、 D^{v} 、 D^{d}$ can be gotten. Using $\mathrm{f}(\mathrm{x}, \mathrm{y})$ to represent the source images as $C_{0}$, Set filter coefficient matrix are respectively $\mathrm{H}$ and $\mathrm{G}$ that corresponding to scaling coefficient $\Phi(x)$ and wavelet coefficient $\Psi(x)$, so two-dimensional wavelet decomposition algorithm can be described as follows:

$$
C_{j+1=\mathrm{H}} C_{j} \mathrm{H}
$$




$$
\begin{aligned}
& D_{j+1=\mathrm{G}}^{h} C_{j} \mathrm{H} \\
& D_{j+1}^{v}=\mathrm{H} C_{j} \mathrm{G} \\
& D_{j+1}^{d}={ }_{\mathrm{G}} C_{j} \mathrm{G}
\end{aligned}
$$

In this function, $J(0,1,2,3 \ldots \ldots . j-1)$ is respectively the layer number of decomposition. $\mathrm{h}, \mathrm{v}, \mathrm{d}$ is respectively the horizontal, vertical and diagonal component. $\mathrm{H}$ and $G_{\text {are }}$ respectively the associate operation matrix of $\mathrm{H}$ and $\mathrm{G}$.

Reconstruction algorithm for wavelet is :

$$
C_{\mathrm{j}-1}=H^{\prime} C_{{ }^{\mathrm{H}}+} G^{\prime} D_{{ }^{\mathrm{H}}+}^{h} H D^{v}{ }_{\mathrm{G}+} G D_{\mathrm{j}}^{d}
$$

$(\mathrm{j}=\mathrm{J}, \mathrm{J}-1, \mathrm{~J}-2 \ldots . .1)$

The more decomposition layers of wavelet there are, the smaller size of image decomposition can be gotten. The pyramid structure is formed after wavelet decomposition. The pyramid structure is also called wavelet decomposition Pyramid. Wavelet transform of image is a kind of multi-scale, multi-resolution image decomposition.

\section{Remote Sensing Image Fusion.}

As a newly emerging technology, the application of remote sensing image fusion technology is promising, and its main purpose is to restore the image data into a new image by analyzing and fusing the data of remote sensing image sent by remote sensor. Remote sensing image fusion has not only realized the composition of the data image, but also strengthened the optimization of image information. The optimization the corresponding image information can be conducted by using certain algorithm. The images obtained by this method are much clearer and more accurate than images obtained by any other single processing method

\section{A. Image fusion based on HIS color transformation}

The specific principles of wavelet algorithm of HIS color transform: (1) Geometrical matching of high resolution images and multispectral images should be made. (2) In order to improve the Explanatory Capability of the multispectral color composition, HIS transform of the multispectral images are needed. (3) Wavelet transform to the high resolution images and the I (luminance) component should be made. (4) The low frequency information of luminance component $\mathrm{I}$ of multispectral image is kept unchanged, and the high frequency information of high resolution image is decomposed by wavelet, then the high frequency information is superimposed on the high frequency component of luminance component I of multispectral image. Inverse wavelets transform is conducted on the luminance component I that has low frequency information and high frequency information. In this way, will the original spectrum information of multi spectral images be retained to the largest extent, and can its spatial resolution be maximized. (5) Finally the H, I, S component transformed are cascaded in RGB three-dimensional space, and then the RGB space image fused is produced. By the above analysis we can see that compared with low resolution image, high resolution image has much edge detail information of the large building and more information of road network. While resolution multispectral image contains rich color information, red represents plants (Band is combined to SPOT NIR band (near infrared band), red band, green band ), grayish-green is for water, brown is for buildings. In general, the main aims of the image fusion are as follows: 1. to improve geometric accuracy of images. 2. to improve the spatial resolution of images. 3. to enhance demonstrating ability. 4 . to improve accuracy of classification. 5. to replace test or repair the related image data.

\section{B. Image fusion based on Wavelet Transform}

Wavelet Transform in image fusion has many advantages. One of the most important advantages is that the image can be decomposed into different frequency domains with Wavelet Transform. As a result, different fusion algorithms can be applied to images in different frequency domains to obtain multi-resolution decomposition of synthetic images, and thus the resolution of source images in different frequency domains are reserved.

\section{The analysis of experimental results}

According to the experimental results, compared with the traditional HIS data fusion algorithm, wavelet transform has the following characteristics

1. Wavelet fusion model can not only select the wavelet basis according to the different characteristics of input image, but assume the detailed information of both sides according to the need of reality. This can achieve better fusion effect and stronger pertinence, and show great practicality.

2. As to the flexibility, in the whole process, Wavelet method can not only complete the band fusion operation, but can complete the multi-band fusion operation. HIS transform can only and must, at the same time, conduct fusion operation on the 3 bands.

3. In comparison, there is much distortion of HIS fusion method in multiresolution data fusion, graveness and chariness is necessary to the data analysis.

\section{The Evaluation of Fused Image Quality}

\section{A. Correlation coefficient $\rho$ and introduction of entropy formula.}

Correlation coefficient $\rho$ is a correlation coefficient between multispectral image and the fused image. With $\rho$, spectrum ability of fusion image can be inspected. High resolution image correlation coefficient $\rho$ can reflect the improvement of the spatial resolution of the fused image.

$$
\rho=\frac{\sum_{i=1}^{m} \sum_{j=1}^{n}(M(i, j)-\bar{M})(F(i, j)-\bar{F})}{\sqrt{\sum_{i=1}^{m} \sum_{j=1}^{n}(M(i, j)-\bar{M})^{2}(F(i, j)-\bar{F})^{2}}}
$$

In this function, $\bar{M}$ and $\bar{F}$ is respectively for mean value $m$ and $n$ which stand for the number of pixels in the 
ranks of the image. An important standard is comentropy of images which is used to measure the richness of image information. Comentropy stands for the average information volume included by image.

According to Shannon information theory, the entropy of an image consisted of 8 bits is:

$$
\mathrm{H}(\mathrm{x})=-\sum P_{I} \log _{2} P_{i}
$$

In function, Pi stands for probability whose gray value of image pixel is $\mathrm{i}$. If the image contains rich information, then $\mathrm{H}(\mathrm{x})$ of the fusion image is higher.

\section{B. The quality evaluation of experimental results}

The correlation coefficient and entropy are adopted to evaluate the effect of image fusion.

The spectrum ability of Wavelet transform fusion can be seen through the correlation coefficient $\rho$ of fused images and multispectral images. By analyzing, we know that the spectrum ability of wavelet transform fusion method is very powerful; considering the influence of resolution, PCA fusion can maximize the image resolution by observing the correlation coefficient $\rho$ of high resolution image and fused image. While wavelet transform fusion method takes the second place. As far as the entropy of fusion images is concerned, wavelet transform fusion and PCA fusion are better. The analysis shows that wavelet transform fusion makes the fused image keep as much multiband spectral information as it can, and it can improve the spatial resolution of the images and balance information content. Wavelet transform fusion method has the obvious superiority, Showed in table 1 .

\section{SUMMARY}

According to the frequency relationship between the multispectral remote sensing image and the high resolution image and the characteristics of wavelet transform, wavelet transform fusion can be carried on. Traditional image fusion methods are PCA method, HIS method and so on. Compared with traditional methods, wavelet transform fusion method not only has a lot of advantages in reserving spectral information, but also has very good fusion effect on improving multispectral influence of space structure and the detail information.

\section{REFERENCES}

[1] WANG Wenjie .Image fusion algorithm based on wavelet transform[J]. China's image journal, 2007, 6A (11): 1130 1135 .

[2] YAN Jingwen. Digital image processing[M].Beijing: National defence industry press, 2007.2:181 182 .

[3] WANG Ailing, YE Mingsheng, DENG Qiuxiang. Technology and application of image processing[M].Beijing: Electronic Industry Press, $2008,1: 362 \sim 365$.

[4] LI Jun, ZHOU Yueqin, LI Deren. The fusion research of the application of wavelet transform in high resolution panchromatic and multi-spectral image[J]. Journal of remote sensing, 1999 (2): 90 97.

[5] WANG Zhijun.The fusion of IKONOS satellite panchromatic image and multispectral image based on Wavelet Theory[J]. Surveying and mapping journal, 2001, 21 (2) : 112 116

TABLE I. FUSION IMAGE QUALITY EVALUATION WITH THE CORRELATION COEFFICIENT AND ENTROPY

\begin{tabular}{|c|c|c|c|c|}
\hline Image & Band number & $\begin{array}{c}\rho \text { (multispectral } \\
\text { image) }\end{array}$ & $\begin{array}{c}\rho \text { (high resolution } \\
\text { image })\end{array}$ & Entropy \\
\hline \multirow{3}{*}{$\begin{array}{c}\text { Multiple spectral } \\
\text { bands }\end{array}$} & 5 & 1 & 0.552 & 7.1148 \\
\cline { 2 - 5 } & 4 & 1 & 0.635 & 6.1206 \\
\cline { 2 - 5 } & 3 & 1 & 0.732 & 6.2997 \\
\hline \multirow{2}{*}{$\begin{array}{c}\text { PCA } \\
\text { Transform fusion }\end{array}$} & 5 & 0.642 & 0.971 & 6.9295 \\
\cline { 2 - 5 } & 4 & 0.812 & 0.827 & 6.1762 \\
\hline \multirow{2}{*}{$\begin{array}{c}\text { Wavelet transform } \\
\text { fusion }\end{array}$} & 3 & 0.740 & 0.946 & 6.7324 \\
\cline { 2 - 5 } & 4 & 0.914 & 0.845 & 6.2719 \\
\cline { 2 - 5 } & 3 & 0.861 & 0.946 & 6.3723 \\
\hline
\end{tabular}

